Check for updates

Cite this: RSC Adv., 2017, 7, 39914

\title{
Influence of melt-draw ratio on the crystalline behaviour of a polylactic acid cast film with a chi structure
}

\begin{abstract}
Ruijie $\mathrm{Xu}^{*}$ Jiayi Xie and Caihong Lei (D) *
Herein, polylactic acid cast films were prepared with different melt-draw ratios via an extrusion casting process. The structure and properties of the prepared films were characterized. The results showed that with the increasing melt-draw ratios (MDRs), an oriented structure appeared at first, and then, a chi structure crystal was formed at higher MDRs. The vertical shish crystals showed a higher melting point than the oriented structure. The PLA molecules were extended by melt-stretching, and weak hydrogen bonds appeared between the carbonyl and methyl bond in the neighbourhood extended PLA molecular chain segments. The hydrogen bond limited the molecular mobility in the amorphous region, which increased the weight fraction of the rigid amorphous region. The stretching-induced crystallization occurred during the melt-stretching process, but the formed crystals were not strictly $\alpha$ form or $\alpha^{\prime}$ form. At lower MDRs, mainly an oriented structure was formed, and with the increase in MDR, meridional streaks in SAXS patterns appeared, accompanying the shish formation. The kebab and oriented structure signals superimposed together; this led to irregular equatorial scattering patterns. In this study, we provide a new insight into PLA crystallization via melt-extrusion cast flow.
\end{abstract}

Received 13th May 2017

Accepted 23rd July 2017

DOI: 10.1039/c7ra05422j

rsc.li/rsc-advances

of the crystalline property changes. These experimental condi-

\section{Introduction}

Polylactic acid (PLA) is a well-known biodegradable semicrystalline polyester that has been widely used in the biomedical, packaging, and agriculture fields. ${ }^{1}$ To date, four types of crystal structures ( $\alpha, \alpha^{\prime}, \beta$, and $\gamma$ forms) have been reported, and many studies have been focused on the structural changes, such as crystallization, crystal transformation, and mesophase formation, of PLA. ${ }^{1-4}$ However, the relatively slow crystallization rate of pure PLA makes it easily quench into the amorphous state during a fast cooling process such as injection, blowing or extrusion moulding. ${ }^{5}$ The enhancement of overall PLA crystallinity is an essential factor to be considered when PLA is utilized for various industrial applications. Many studies have been carried out to enhance the overall crystallization rate by adding nucleators, such as talc, phthalimide, and triphenyl phosphate. $^{6,7}$ Some studies show that blending of poly(L-lactide) (PLLA) and poly(D-lactide) (PDLA) is effective for controlling the overall crystallinity and obtaining the stereocomplex crystal with a higher melting point. ${ }^{8}$ Secondary process technology, such as annealing or stretching, can also increase the nucleation density and improve the crystallinity. All the abovementioned studies are based on the static crystallization process or only stretching of amorphous films for investigation

Guangdong Provincial Key Laboratory of Functional Soft Condensed Matter, School of Materials and Energy, Guangdong University of Technology, Guangzhou 510006, P. R. China.E-mail:xu565786@163.com; lch528@gdut.edu.cn tions are different from those of the fiber fabrication process, where the elongation stress field is applied after the materials leave the extruder die.

Several studies about the PLA melt-spinning process showed that the glass transition temperature $T_{\mathrm{g}}$, melting point $\left(T_{\mathrm{m}}\right)$, and crystallinity were considerably increased depending on the melt-draw ratio (MDR). Cicero et al. ${ }^{9}$ found that $T_{\mathrm{g}}$ increased from $61.7^{\circ} \mathrm{C}$ to $68.7^{\circ} \mathrm{C}$, whereas the crystallinity reached $51 \%$ at an MDR value of 8 . Schmack et al. ${ }^{\mathbf{1 0}}$ observed that the fibers spun at $1000 \mathrm{~mm} \mathrm{~min}^{-1}$ remained noncrystalline, and partial crystallization occurred at a spinning velocity above $2500 \mathrm{~mm}$ $\min ^{-1}$. At a higher spinning temperature and stretching velocity, PLA fibers with $\beta$ crystals were formed. ${ }^{11}$

Besides the melt-spinning process, melt-stretching is another important method where the polymer melt is stretched during the crystallization process. This method has been widely used to fabricate polypropylene, polyethylene, polyvinylidene fluoride, and poly(4-methyl-1-pentene) precursor films with row-nucleated crystalline structures. ${ }^{12-15}$ With the increase of melt-draw ratios, the crystallinity and orientation are enhanced.

During the melt-stretching extrusion process, the polymer molecules are stretched at the exit of the extruder die and orient along the flow direction. The high molecular weight chains will form stable oriented nuclei (shish) and the other chains create the chain-folded lamellae over these nuclei sites. This process will effectively increase the crystallization velocity. ${ }^{16}$ It is noted that the stretching velocity and melt-draw ratio herein are much 
smaller than those obtained during the spinning process. Usually, the stretching velocity in melt-extrusion cast is below $10 \mathrm{~s}^{-1}$, whereas that of spinning is more than hundred meters per second. The crystallization behaviour and morphology were different from those achieved during the melt-spinning process.

Depending on the melt-extrusion conditions, a large amount of PLA molecules remains uncrystallized when the molecules are stretched and cooled between the die and chill roll. Then, upon subsequent crystallization, the non-crystalline PLA chains become trapped between the growing crystals inside or outside of the lamella stacks. As a result of the highly entangled nature of the PLA chains, the segmental movement of the amorphous PLA chains becomes restricted. The concept of a third phase, termed as the rigid amorphous fraction (RAF), was introduced to explain the constraints exerted by the crystals upon the mobility of the amorphous phase. ${ }^{17}$ The existence of RAF shows no contribution to the heat capacity increase at the glass transition $T_{\mathrm{g}}$ and to the heat of fusion of the crystals. ${ }^{\mathbf{1 8 , 1 9}}$

On the other hand, a mesophase has been reported in oriented polymer films and fibers. ${ }^{20,21}$ The mesophase indicates an oriented phase fraction, which exists as a transition layer, or an interface with a medium level of order between crystalline and amorphous regions, or an intermediate state between the crystalline and amorphous states. The physical nature of this state of intermediate order has been interpreted controversially. It has been proposed that the mesophase can be a type of paracrystalline order, or conformationally disordered structure. ${ }^{22-24}$

For polymers with a slow crystallization velocity, a complex structure composed of soft amorphous fraction (SAF), RAF, mesophase fraction, and crystal fraction would be obtained via flow-induced crystallization. Therefore, it is necessary to explore the complex crystalline structure prepared by the melt-extrusion cast process. The orientation structure and properties still need further studies. In this study, the PLA cast extrusion films were prepared at different melt-draw ratios between the cast roll and extruder. The structure and properties of the PLA cast films were studied.

\section{Experimental}

\section{Material}

Polylactic acid (4043D) with a melt flow index of $9.8 \mathrm{~g} / 10 \mathrm{~min}$ $\left(190{ }^{\circ} \mathrm{C}, 2.16 \mathrm{~kg}\right)$ from NatureWorks, USA, was used. The number- and weight-average molecular weights were $4.05 \times 10^{5}$ and $5.47 \times 10^{5}$, respectively, as evaluated by gel permeation chromatography (Viscotek 270max) using polystyrene as the standard and tetrahydrofuran as the solvent. The glass transition temperature of amorphous PLA is at $59{ }^{\circ} \mathrm{C}$ with a change in heat capacity of $0.44 \mathrm{~J} \mathrm{~g}^{-1}$. For $100 \%$ crystalline PLA, the heat of fusion is estimated to be $93 \mathrm{~J} \mathrm{~g}^{-1}$ at a peak melting temperature of $T_{\mathrm{m}}=153.7^{\circ} \mathrm{C}^{25}$

\section{Film preparation}

The cast films were prepared by extrusion casting through a Tslot die. During extrusion, uniaxial (machine direction, MD) stretching was applied to the PLA melt. The die temperature was set at $200{ }^{\circ} \mathrm{C}$, and the melt-draw ratios (MDRs) were set at 40,80 , $107,134,152,177$, and 211 . MDR is a specific value between the line speed of the chill roll and the extrudate velocity at the exit of the die. The films were produced at a chill roll temperature of $50{ }^{\circ} \mathrm{C}$ (Fig. 1).

\section{Film characterization}

Atomic force microscopy (AFM). Atomic force microscopy (AFM) analysis was carried out using a Solver P47-Pro instrument with silicon AFM cantilevers (NSG10, NT-MET, Russia). The spring constants of the cantilevers were between 5.5 and $22.5 \mathrm{~N} \mathrm{~m}^{-1}$, and the resonance frequencies were between 190 and $325 \mathrm{kHz}$.

Differential scanning calorimetry (DSC). Differential scanning calorimetry (DSC) measurements were carried out via a DSC Q2000 (TA Instruments, USA) using nitrogen as a purge gas. The temperature and heat flow were calibrated with indium. The DSC trace was obtained upon heating at a rate of $10{ }^{\circ} \mathrm{C} \min ^{-1}$. The crystallinity was calculated as follows:

$$
\text { Crystallinity }(\%)=\frac{\Delta H_{\mathrm{m}}-\Delta H_{\mathrm{cc}}}{\Delta H_{\mathrm{m}}^{0}} \times 100 \%
$$

where $\Delta H_{\mathrm{m}}$ is the endothermic heat of melting, $\Delta H_{\mathrm{cc}}$ is the exothermic heat of cold-crystallization, and $\Delta H_{\mathrm{m}}^{0}$ is the heat of melting of perfectly crystalline PLA $\left(93 \mathrm{~J} \mathrm{~g}^{-1}\right) .{ }^{26}$

Wide-angle X-ray diffraction (WAXD). Wide-angle X-ray diffraction (WAXD) measurements were carried out using a Bruker AXS X-ray goniometer (Germany). The generator was set up at $40 \mathrm{kV}$ and $40 \mathrm{~mA}$, and the copper $\mathrm{Cu} \mathrm{K} \alpha$ radiation was selected using a graphite crystal monochromatic. The sample to detector distance was fixed at $8 \mathrm{~cm}$ with the scan direction parallel to the extrusion-stretching direction.

Fourier transform infrared (FTIR) spectroscopy. Fourier transform infrared (FTIR) spectroscopy spectra were obtained using a Nicolet Magna is50 FTIR instrument from Thermo Electron Corp. (DTGS detector, resolution 4 per $\mathrm{cm}$, accumulation of 128 scans). The beam was polarized via a Spectra-Tech zinc selenide wire grid polarizer from Thermo Electron Corp.

Small angle X-ray scattering (SAXS) experiments. Small angle $\mathrm{X}$-ray scattering (SAXS) experiments were performed using synchrotron radiation with $\lambda=0.154 \mathrm{~nm}$ at Beamline $1 \mathrm{~W} 2 \mathrm{~A}$ of the Beijing Synchrotron Radiation Facility (Beijing, China). Mar165-CCD was set at a $5000 \mathrm{~mm}$ sample-detector distance in the direction of the beam for small angle X-ray scattering (SAXS) data collection.

Scanning Electronic Microscopy (SEM). For observation of the crystalline morphology, the samples were etched in a watermethanol ( $1: 2$ by volume) solution containing $0.025 \mathrm{~mol} \mathrm{~L}^{-1}$ of

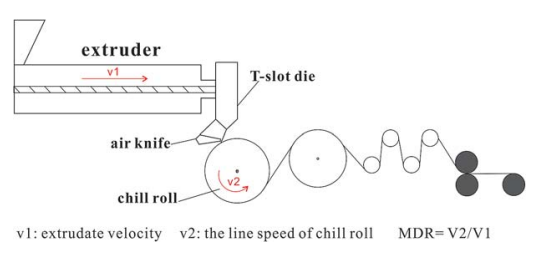

Fig. 1 The schematic for processing cast films. 
sodium hydroxide for $24 \mathrm{~h}$ at $25{ }^{\circ} \mathrm{C}$. Subsequently, the etched surface was cleaned with distilled water followed by ultrasonication. An SEM (S3400N, Hitachi, Japan) was utilized for all samples, which were sputter-coated with gold before observations, and the accelerated voltage was held at $10 \mathrm{kV}$.

\section{Results and discussion}

\section{Effects of melt-draw ratios on the crystalline structure of cast} films

The height images of the surface morphology for the extrusion cast films with different MDRs were obtained using AFM and are shown in Fig. 2. The height difference sections along line A and line $\mathrm{B}$ in the extrusion direction are also included. It can be seen that at lower MDR, some oriented structures along the machine direction (MD) appear. With the increasing MDR value, many new textures grow perpendicularly to the oriented initial structure on its both sides. Compared to that in line A, the obvious height difference appears along the machine direction in line B. The orientation-induced crystallization induces the appearance of a vertical structure. These newly grown crystals gradually become pronounced with the increase in MDR. The interesting phenomenon is the formation process of this kind of chi structure. First, some oriented structures appear, and then, a perpendicular structure is induced. A similar crossed crystallization structure formation process has been reported by Murase et al. ${ }^{27}$ during spinning, where the string-like structure appeared at first via flow-induced phase separation, and then, a shish-kebab structure was formed via a self-assembly process. Analogically, during melt-stretching, the initial oriented structure is first formed and then separated from the matrix because of the viscosity difference. Then, with the increase in the melt-stretching velocity, the oriented structure affords nuclei for the growth of the vertical structure. When we enlarged the vertical structure, we noticed that some analogous kebab structures appear. Compared with the shishkebab structure formed during melt-spinning, the vertical structure turns longer and distributes sparsely in our experiment. The main reason is the huge stretching rate difference between the melt-stretching and melt-spinning. Ma et al. ${ }^{17}$ indicated that during the electrospun process, the moved (soft)

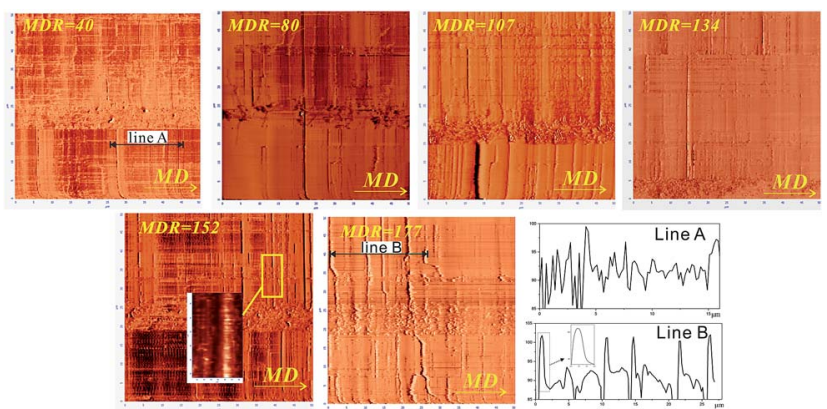

Fig. 2 AFM height images revealing the surface morphology of the extrusion cast film with different MDRs and the height difference sections along line $A$ and line $B$. The image size is $50 \times 50 \mu \mathrm{m}$. The machine direction is horizontal.

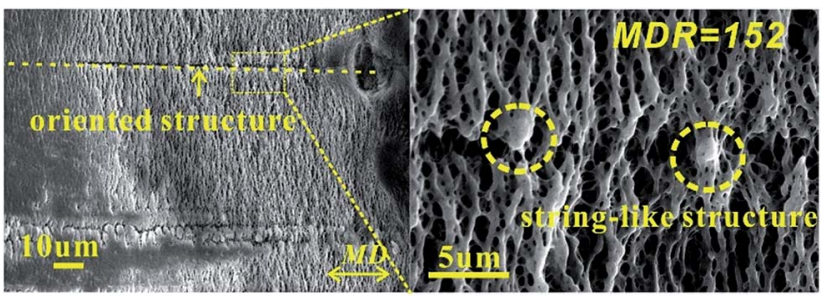

Fig. 3 SEM observation of crystalline morphology of the cast film with an MDR value of 152

amorphous phase, rigid amorphous phase, mesophase, and crystal coexisted in the system. Moreover, a similar structure may exist during melt-stretching. The relationship between the initial oriented structure and newly grown crystals has been discussed hereinafter.

The etched surface of the PLA cast film with an MDR value of 152 is shown in Fig. 3. It is interesting that the oriented parts are totally etched, and the lamellar structure kebab crystals are reserved. The string-like structure is also saved after etching. This result means that the string-like structure is closer to the crystalline structure. The distance of the neighbourhood-like kebab crystal is about 3-5 $\mu \mathrm{m}$, which is similar as that of the AFM structure. This stacked structure has also been reported by $\mathrm{Xu}$ et al. ${ }^{42}$ under shear field.

Fig. 4 shows the typical FT-IR spectra of the PLA films with different MDRs. Some distinct peaks within the regions of $2858-2995 \mathrm{~cm}^{-1}$ correspond to the asymmetrical and symmetrical stretching mode of the $\mathrm{CH}_{3}$ and $\mathrm{CH}_{2}$ groups. The fundamental modes of the carbonyl vibration occur in the region 1751-1758 $\mathrm{cm}^{-1}$. It should be noted that upon close examination, the spectra reported by the authors are largely overabsorbed in the thick samples. Moreover, the intensity of the bands near $3700-3450 \mathrm{~cm}^{-1}$ is dependent on the degree of overabsorption. The range between 3700 and $3450 \mathrm{~cm}^{-1}$ is due to an overtone mode, whose intensity increases with the degree of over-absorption of its corresponding fundamental modes. The characteristic spectra of $\mathrm{C}-\mathrm{O}-\mathrm{C}$ occur in the region 1050-1200 $\mathrm{cm}^{-1}$. The peaks in the region $1430-1480 \mathrm{~cm}^{-1}$ represent $\mathrm{C}-\mathrm{H}$

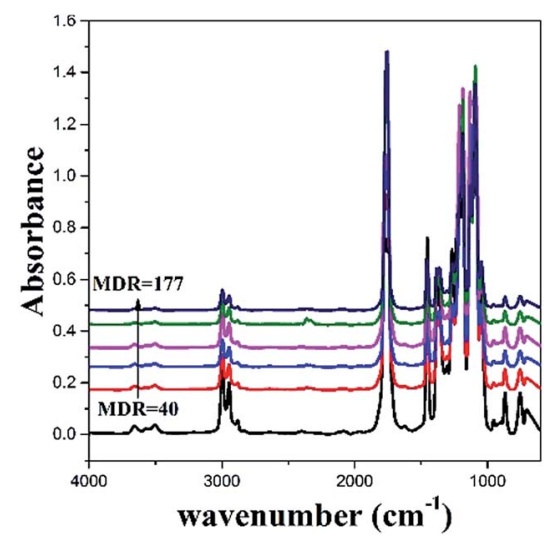

Fig. 4 FT-IR spectra curves of the PLA films prepared at different MDRs. 
deformation, whereas those in the region $733-756 \mathrm{~cm}^{-1}$ represent the skeletal vibration of the methylene groups.

To discuss the effect of melt-stretching on PLA crystal structure transformation, the characteristics of the local regions were followed. As Opaprakasit et al. ${ }^{28}$ mentioned, bands near $3500 \mathrm{~cm}^{-1}$ were related to the weak $\mathrm{C}=\mathrm{O}$ bond stretching, implying $\mathrm{C}=\mathrm{O}$ bond elongation due to a specific reaction, especially hydrogen bonding. The enlarged spectra and their second derivatives are shown in Fig. 5. With the increasing MDR, the peak at $3500 \mathrm{~cm}^{-1}$ becomes wide and weak. Moreover, two distinct peaks located at 3527 and $3504 \mathrm{~cm}^{-1}$ in second derivatives were observed, which belonged to the amorphous and crystalline region, respectively. With an enhancement in melt-stretching, the $\mathrm{C}=\mathrm{O}$ bond elongation in the amorphous region is reduced and turns strong in the crystalline region; this suggests a weak interaction between the carbonyl and methyl bond in the neighbourhood extended PLA molecular chain segments to form the ordered structure. Compared to Opaprakasit's result, the crystalline $\mathrm{C}=\mathrm{O}$ overtone found in the pure PLLA is roughly located at $30 \mathrm{~cm}^{-1}$ higher than that of its stereocomplex, reflecting a strong $\mathrm{C}=\mathrm{O}$ bond. ${ }^{28}$

Fig. 6 shows the IR spectra in the range between 1000 and $650 \mathrm{~cm}^{-1}$ and the second derivatives spectra between 950 and $900 \mathrm{~cm}^{-1}$ with different MDRs. As abovementioned, the bands at $707,755,869$, and $956 \mathrm{~cm}^{-1}$ are attributed to the amorphous region. With the increasing $\mathrm{MDR}$, the band at $869 \mathrm{~cm}^{-1}$, which is assigned to the $\mathrm{C}-\mathrm{C}$ backbone stretching $[\nu(\mathrm{C}-\mathrm{COO})]$ is shifted to a higher wavenumber of $871 \mathrm{~cm}^{-1}$ because of the dipoledipole interaction in the crystal lattice. ${ }^{29}$ Moreover, the weak shoulder peaks at 916,739 , and $680 \mathrm{~cm}^{-1}$ are observed. They are believed to be relative to the crystalline structure formation.

In the backbone vibration region $\left(970-730 \mathrm{~cm}^{-1}\right.$, assigned to skeletal C-C stretching coupled with the $\mathrm{CH}_{3}$ rocking mode), ${ }^{30}$ the melt-stretched PLA films gives a band at $916 \mathrm{~cm}^{-1}$, whereas there is no band around $920 \mathrm{~cm}^{-1}$ for the amorphous PLLA. It has been found that the band at $920 \mathrm{~cm}^{-1}$ is related to the ordered helical chain conformation of PLLA. ${ }^{31}$ Stoclet et al. ${ }^{32}$ and Zhang et al. ${ }^{33}$ reported that the band at $918 \mathrm{~cm}^{-1}$ belongs to the mesophase formed in the quench- or draw-induced samples. However, during the extrusion cast process, the helix
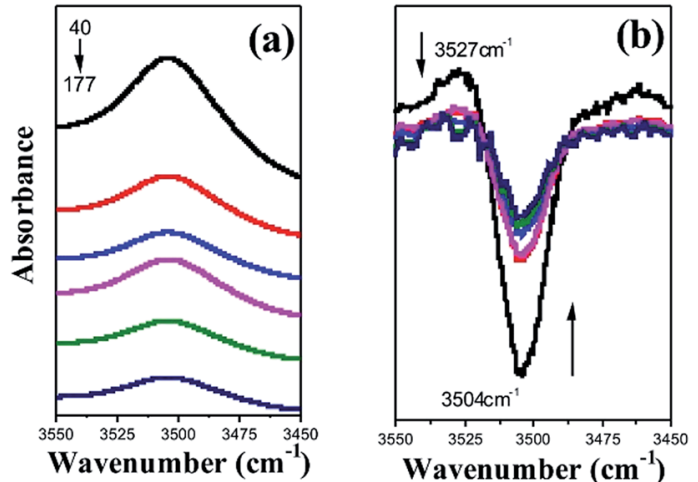

Fig. 5 The enlarged spectra near $3500 \mathrm{~cm}^{-1}$ and their second derivatives.

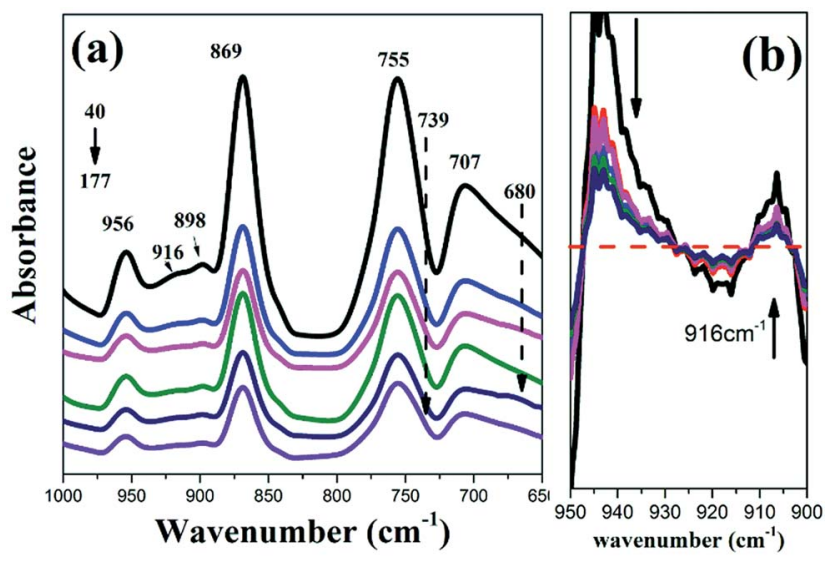

Fig. 6 IR spectra (a) and the second derivatives at $960-900 \mathrm{~cm}^{-1}(\mathrm{~b})$ of the PLA cast films with different MDRs.

conformation is weak and imperfect. The characteristic peak shifts to a lower wavenumber. Therefore, this may be the first report on the observation of a conformational band at $916 \mathrm{~cm}^{-1}$ for PLLA.

The different melt-draw ratios show a remarkable influence on the molecular orientation. For quantitative comparison, the dichroic ratio $R$ and structural absorbance $A$ were determined using the following relations: ${ }^{20}$

$$
\begin{gathered}
R=A_{\|} / A_{\perp} \\
A=\left(A_{\|}+2 A_{\perp}\right) / 3
\end{gathered}
$$

where $A_{\|}$and $A_{\perp}$ are the parallel and perpendicular absorbance of the desired absorption band, respectively.

Fig. 7 compares the dichroic ratio $R$ of the 916 and $956 \mathrm{~cm}^{-1}$ band, which are assigned to the oriented primary crystal phase and amorphous phase, respectively. With the increasing meltdraw ratio, the orientation fraction improves in both the primary crystal phase and amorphous phase. However, the molecular orientation in the amorphous phase becomes much

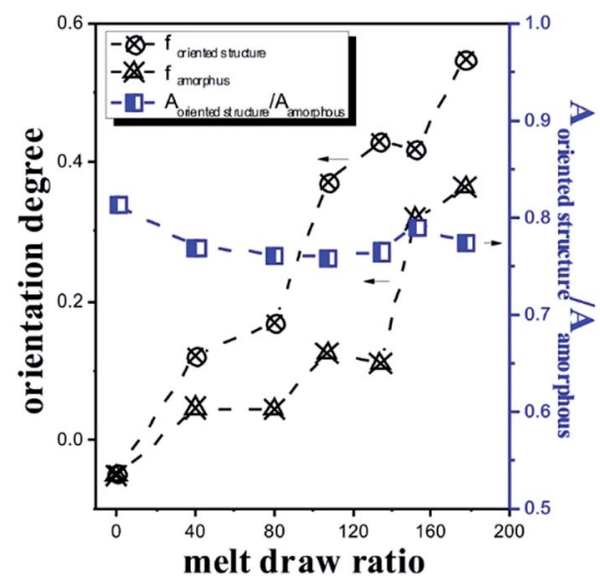

Fig. 7 Molecular orientation and relative orientation content of PLA cast films with different MDRs. 
lower than that in the crystalline phase. Moreover, two possible reasons could account for it. First, it could result from the different extensibility limit of segments trapped among entanglement junctions since further increase in the molecular orientation will be delayed, whereas segments will become welloriented. ${ }^{34}$ The main difference in the oriented primary crystal and amorphous phase is the degree of molecule chain entanglement. The entanglement limits significant segmental extensibility and the orientation and crystallization retard the molecule segmental relaxation. The second possibility could be related to higher intermolecular cohesion in the oriented primary crystal phase than that in the amorphous phase beyond the segmental extensibility limit, which can alleviate relative slippage among stretched segments and thus benefit the increase in molecule orientation. $\mathrm{Lv}$ et al. $^{\mathbf{2 0}}$ proved that the cohesive structure required remarkable conformational ordering to reduce steric hindrance. The relative orientation content between the oriented crystal and amorphous phases is also shown in Fig. 7. The value almost remains constant because of synchronized stretching in the oriented primary crystal and amorphous phase in the melt state.

Based on the FTIR result, we believe that an incomplete primary crystal is formed during melt stretching, derived from the weak hydrogen bond between the extended PLA molecular chain segments. To more carefully study the crystal structure, the WAXD profiles of the PLA cast films with different MDRs from 40 to 177 are given in Fig. 8. Sharp crystalline peaks are observed at the $2 \theta$ values around $21.4^{\circ}, 23.8^{\circ}$, and $26.6^{\circ}$ for all samples. These three peaks are attributed to the diffraction from the (210), (015), and (207) lattice planes. The (210) lattice plane is the characteristic of an $\alpha$ crystal reported by Zhang et $a l .{ }^{35} \mathrm{Cho}^{36}$ found that when the annealing temperature was higher than $155{ }^{\circ} \mathrm{C}$, the 015-reflection appeared and it was attributed to the transformation of the disordered part into crystals during heating. Herein, the existence of the 015-reflection indicates the formation of ordered crystals during melt stretching. However, it is surprising that the main reflection peak of $\alpha$ or $\alpha^{\prime}$ form crystals at $16.8^{\circ}$ for both (200) and (110)

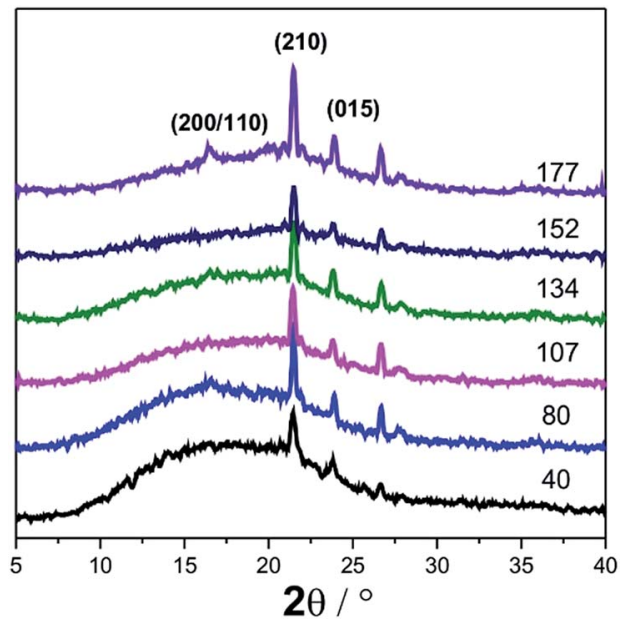

Fig. 8 WAXD curves of the PLA cast films with different MDRs. reflections does not appear until the MDR is 177 . We believe that during melt stretching, the molecular orientation induces the formation of crystal nuclei and some weak or imperfect primary crystals. However, these crystals are not strictly $\alpha$ form or $\alpha^{\prime}$ form. This result is in agreement with the FTIR spectra, where the new characteristic peak at $916 \mathrm{~cm}^{-1}$ corresponds to this incomplete primary crystal.

Fig. 9 shows the DSC curves of PLA films with different MDRs. The enthalpy value of each crystallization peak and the crystallinity of the samples are listed in Table 1 . All the curves exhibit a glass transition temperature $\left(T_{\mathrm{g}}\right)$, a broad coldcrystallization exotherm $\left(T_{\mathrm{cc}}\right)$, and two melting peaks $\left(T_{\mathrm{m} 1}\right.$ and $T_{\mathrm{m} 2}$ ). The samples prepared at the MDR values of 40 and 80 show approximate DSC curves. The $T_{\mathrm{g}}$ does not change much and the $T_{\mathrm{cc}}$ shifts to lower temperatures and becomes sharper with the increase in MDR from 80 to 152 . This may be since the melt-stretching process enhances the molecular orientation and the formation of the initial crystalline nuclei for coldcrystallization. Similar results have been reported by Lee et $a{ }^{37,38}$ Further increase in the MDR to 177 results in the movement of $T_{\mathrm{g}}$ to lower temperatures. The relaxation peak almost disappears. $T_{\text {cc }}$ becomes broad and moves to higher temperatures because of a reduction of the initial crystalline nuclei numbers. During the melt stretching extrusion process, the stretching-induced crystallization only occurs within the limited space between the die and the chill roll. Higher MDR shortens the staying time within this space, and there is not enough time for nuclei formation; this results in lower crystallinity.

The melting point and crystallinity of the samples are shown in Fig. 10. The phenomenon of double melting peaks in the PLA cast film has been reported by Zhang et al., ${ }^{31}$ but they did not provide the reason for it. We notice that the $T_{\mathrm{m} 1}$ peak becomes weak and the $T_{\mathrm{m} 2}$ peak is obviously enhanced with an increase in MDR. The abovementioned AFM images show that at lower MDRs, the oriented structure is mainly induced, and then, more vertical crystals (Chi-structure) are formed at higher MDRs. Then, it is deduced that at lower MDRs, $T_{\mathrm{m} 1}$ originates from the melting of the oriented structure and $T_{\mathrm{m} 2}$ is related to the

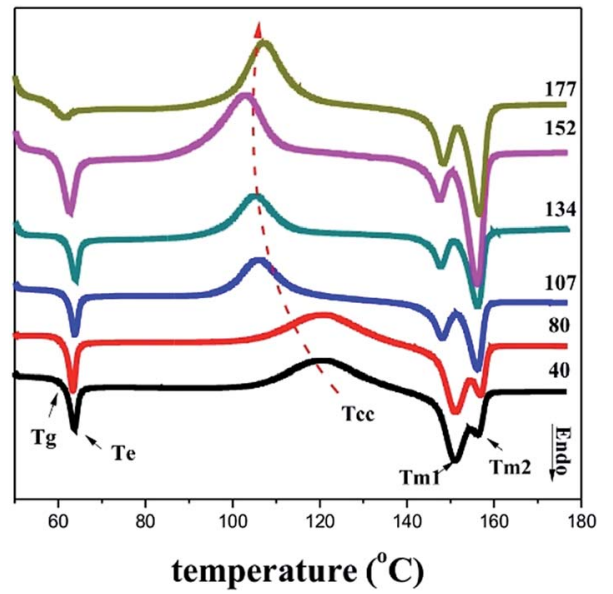

Fig. 9 DSC curves of the PLA films prepared at different MDRs. 
Table 1 The enthalpy value of each crystallization peak and the crystallinity of each sample

\begin{tabular}{lrrccrr}
\hline MDR & \multicolumn{1}{c}{40} & \multicolumn{1}{c}{80} & 107 & 134 & 152 & 177 \\
\hline$H_{\mathrm{C}}\left(\mathrm{J} \mathrm{g}^{-1}\right)$ & 36.3 & 36.9 & 37.1 & 43.8 & 43.1 & 41.0 \\
$H_{\mathrm{M}}\left(\mathrm{J} \mathrm{g}^{-1}\right)$ & 38.5 & 41.2 & 42.5 & 49.6 & 51.3 & 47.6 \\
$X(\%)$ & 2.3 & 4.6 & 5.82 & 6.21 & 8.8 & 7.1
\end{tabular}
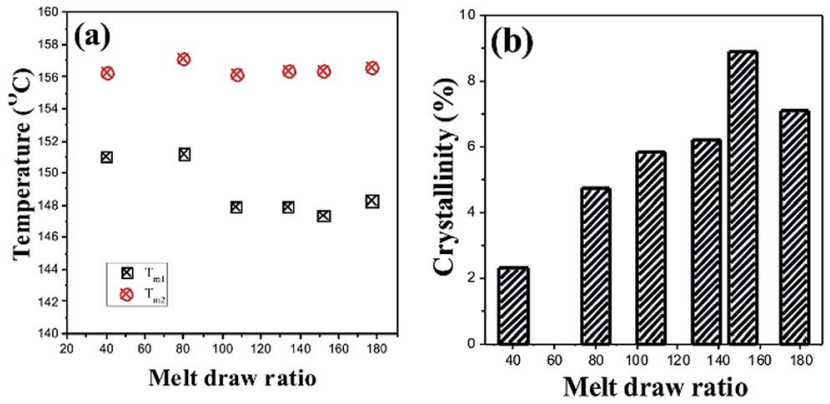

Fig. 10 Melting temperature and crystallinity of the PLA films prepared at different MDRs.

vertical crystals. With the increasing MDR, the more initially oriented structure is transformed into a higher ordered crystalline structure, and more vertical crystals are induced; this leads to the improvement of the $T_{\mathrm{m} 2}$ peak. We also notice that the melting temperature of the cast film is $10{ }^{\circ} \mathrm{C}$ lower than that of the normal PLLA crystalline samples ${ }^{33,35}$ because of the weak crystalline structure. Although the weak hydrogen bond between the carbonyl and methyl bond in the neighbourhood extended PLA molecular chain segments could increase the melting temperature, the efficiency was limited. The crystal formed in the melt-stretching process is still very weak and incomplete. The crystallinity shows the same trend. When the MDR is smaller than 152, the crystallinity is increased from $2.3 \%$ to $8.9 \%$. Upon further increasing the MDR, the crystallinity decreases to $7.8 \%$. Apparently, fast stretching shows no benefit on the force-induced crystallization for polymers with slow crystalline velocity.

For crystals with slow crystallization velocity, a soft amorphous region (SA), rigid amorphous region (RA), and crystalline region (C) coexist. The weight fractions $\Phi_{\mathrm{I}}$ for I $=\mathrm{SA}$, RA, and C of these three phases can be determined by DSC. ${ }^{17} \Phi_{\mathrm{C}}$ is equivalent to crystallinity, and $\Phi_{\mathrm{SA}}$ is calculated as follows:

$$
\Phi_{\mathrm{SA}}=\Delta C_{\mathrm{p}} / \Delta C_{\mathrm{p} 0}
$$

where $\Delta C_{\mathrm{p} 0}$ is the heat capacity increase at the glass transition for $100 \%$ amorphous polymer. Herein, for PLA, $\Delta C_{\mathrm{p} 0}$ is $0.61 .{ }^{17}$ The weight fraction of the rigid amorphous region is calculated as follows:

$$
\Phi_{\mathrm{RA}}=1-\Phi_{\mathrm{c}}-\Phi_{\mathrm{SA}}
$$

As seen in Table 2, $\Phi_{\mathrm{SA}}$ decreases with the increasing MDR. The $\Phi_{\mathrm{SA}}$ is decreased to 0.63 for the melt-extrusion cast film produced using an MDR of 152; this indicates that 0.149 RAF
Table 2 Soft amorphous, rigid amorphous, and crystalline fractions of melt-extrusion cast films with different MDRs

\begin{tabular}{lllllll}
\hline MDR & 40 & 80 & 107 & 134 & 152 & 177 \\
\hline$\Phi_{\mathrm{C}}$ & 0.023 & 0.047 & 0.058 & 0.062 & 0.089 & 0.071 \\
$\Phi_{\mathrm{SA}}$ & 0.95 & 0.83 & 0.77 & 0.75 & 0.63 & 0.78 \\
$\Phi_{\mathrm{RA}}$ & 0.027 & 0.123 & 0.172 & 0.188 & 0.281 & 0.149
\end{tabular}

exists in this sample. An endothermic peak is also observed just above $T_{\mathrm{g}}$ in the DSC curves (Fig. 8). Wunderlich pointed out that for any ordering connected with the RAF, a decrease in enthalpy would be expected, whereas disordering would yield an endotherm. ${ }^{39}$ The decrease in heat capacity increment at $T_{\mathrm{g}}$ confirms that RAF is able to exist in the films. The weak hydrogen bond between the carbonyl and methyl bond may be the main reason affecting the molecular mobility in the amorphous region.

To better understand how the crystals are formed, the cast films with different MDRs were tested via SAXS experiments. The extrusion is in the vertical direction as indicated by the arrow in Fig. 11. At an MDR value of 80, only two sharp SAXS patterns on the equatorial direction are observed. No pronounced vertical patterns can be found. This is in agreement with the former DSC results, where the $T_{\mathrm{m} 1}$ peak is stronger than that of $T_{\mathrm{m} 2}$. The equatorial pattern is attributed to the oriented crystalline structure induced by low MDR stretching, similar to that obtained for lower shear rate shearing-only induced layered structure. ${ }^{40}$ These results further prove the formation of a mainly oriented crystalline structure at lower MDRs. With the increase in MDR to 134 and 177, the scattering intensity in the equatorial direction is not uniform and becomes weak obviously; a strong scattering pattern appears in the meridional direction, in agreement with the abovementioned vertical crystal formation at higher MDRs. As shown in the AFM images, a multilevel-kebab structure appears at a higher MDR. The meridional pattern is contributed by vertical shish crystals and the irregular scattering pattern on the equatorial direction is the superimposed signals of the oriented initial crystals and the grown kebab. The SAXS pattern transformation process is similar to the change of the shish nuclei model during the flow-induced crystallization process. ${ }^{41}$

A schematic of the crystalline structure formation process is shown in Fig. 12. At a low melt-draw ratio, the crystallinity is very low. The soft amorphous region and rigid amorphous region (oriented structure) coexist in the system. With the increasing melt-draw ratio, the multilevel-kebab structure is observed, but these crystals are not strictly $\alpha$ or $\alpha^{\prime}$ form crystals.

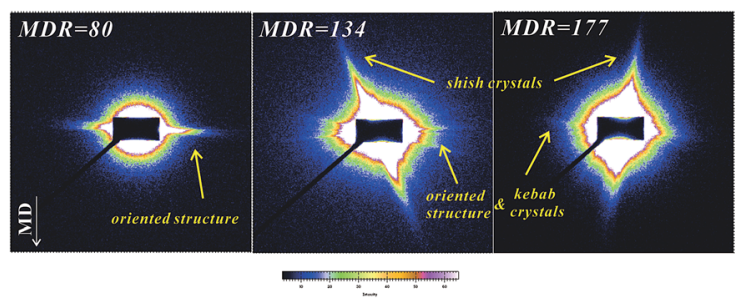

Fig. 11 2D-SAXS patterns of the cast films with different MDRs. 

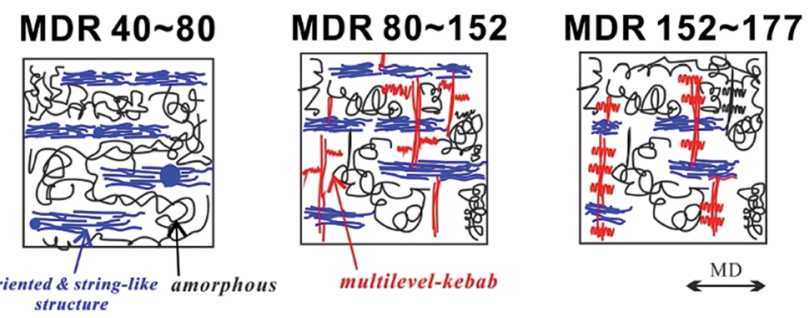

Fig. 12 The schematic of structural evolution.

When the MDR is increased to 177 , the multilevel-kebab structure turns intensive. The rigid amorphous region turns discontinuous due to the fast crystalline velocity induced by the higher melt-stretching field.

\section{Conclusions}

The structure and crystalline properties of PLA extrusion cast film with different melt-draw ratios were described based on the AFM, FTIR, DSC, WAXD, and 2D-SAXS data. The oriented crystalline structure forms at lower melt-draw ratios, and higher melt-draw ratios induce pronounced chi structure crystals. The DSC curves show that all the samples have two melting peaks, corresponding to the chi structure. The melting point of the oriented structure is near $149^{\circ} \mathrm{C}$, and the vertical shish crystals have a higher melting point at $156^{\circ} \mathrm{C}$. The melting point is $10^{\circ} \mathrm{C}$ lower than that of a normal PLA spherulite because of the weak hydrogen bond. The crystallinity is gradually improved with the increasing MDR from 40 to 152 and obviously reduced at higher MDRs. The melt-stretching-induced crystals are not strictly an $\alpha$ form or $\alpha^{\prime}$ form, and the characteristic bond of the initial oriented structure is at $916 \mathrm{~cm}^{-1}$. At lower MDRs, the chain segments are oriented and stretched. At higher MDRs, the chistructure crystals appear on both sides of the initial oriented crystalline structure. This melt stretching process typically increases the rigid amorphous region fraction.

\section{Acknowledgements}

The authors would like to thank the National Science Foundation of China under Grant No. 51603047, Guangdong Province Major Key Projects of Applied Research and Development of Science and Technology (2015B090925021), Guangzhou Science and Technology Plan Project (201510010037), Guangdong Province Ordinary University Innovation Project, the PhD Startup Fund of the Natural Science Foundation of Guangdong Province, China (2016A030310344), and China Postdoctoral Science Foundation funded project (2016M592461) for financial support. We would also like to thank Shenzhen Senior Materials Company, Ltd., for generously supplying the raw materials.

\section{Notes and references}

1 M. Peddy, S. Vivekanandhan, M. Misra, S. Bhatiac and A. Mohanty, Prog. Polym. Sci., 2013, 38, 1653.
2 L. Bouapao, H. Tsuji, K. Tashiro, J. Zhang and M. Hanesaka, Polymer, 2009, 50, 4007.

3 J. Kalish, K. Aou, X. Yang and S. Hsu, Polymer, 2011, 52, 8141.

4 G. Stoclet, R. Seguela, J. Lefebvre and M. Vert, Macromolecules, 2011, 44, 4961.

5 Z. Gui, C. Lu and S. Cheng, Polym. Test., 2013, 32, 15.

6 H. Xiao, P. Li, X. Ren, T. Jiang and J. Yeh, J. Appl. Polym. Sci., 2010, 118, 3558.

7 D. He, Y. Wang, C. Shao, G. Zheng, Q. Li and C. Shen, Polym. Test., 2013, 32, 1088.

8 J. Sun, J. Shao, S. Huang, B. Zhang, G. Li, X. Wang and X. Chen, Mater. Lett., 2012, 89, 169.

9 J. Cicero, J. Dorgan, J. Janzen, J. Garrett, J. Runt and J. Lin, J. Appl. Polym. Sci., 2002, 86, 2828.

10 G. Schmack, B. Tändler, R. Vogel, R. Beyreuther, S. Jacobsen and H. Fritz, J. Appl. Polym. Sci., 1999, 73, 2785.

11 B. Gupta, N. Revagade, N. Anjum, B. Atthoff and J. Hilborn, J. Appl. Polym. Sci., 2006, 100, 1239.

12 C. Lei, S. Wu, Q. Cai, R. Xu, B. Hu and W. Shi, Polym. Int., 2014, 63, 584.

13 B. Hu, C. Lei, R. Xu, W. Shi, Q. Cai, H. Mo and C. Chen, J. Plast. Film Sheeting, 2013, 30, 300.

14 B. Hu, C. Lei, R. Xu, W. Shi, Q. Cai, H. Mo and C. Chen, J. Plast. Film Sheeting, 2015, 31, 78.

15 A. Ma, L. Xu, B. Yin, M. Yang and B. Xie, $R S C A d v ., 2016,6$, 62038.

16 R. Lv, R. Na, Z. Li, S. Zou, N. Tian, F. Chen and Q. Fu, J. Macromol. Sci., Part B: Phys., 2011, 50, 2042.

17 Q. Ma, M. Pyda, B. Mao and P. Cebe, Polymer, 2013, 54, 2544.

18 R. Androsch and B. Wunderlich, Polymer, 2005, 46, 12556.

19 W. Qiu, A. Habenschuss and B. Wunderlich, Polymer, 2007, 48, 1641.

20 R. Lv, B. Na, N. Tian, S. Zou, Z. Li and S. Jiang, Polymer, 2011, 52, 4979.

21 A. Sanz, A. Nogales, T. Ezquerra, M. Soccio, A. Munari and N. Lotti, Macromolecules, 2010, 43, 671.

22 S. Ran, X. Zong, D. Fang, B. Hsiao, B. Chu and R. Phillips, Macromolecules, 2001, 34, 2569.

23 S. Bruckner, S. Meille, V. Petraccone and B. Pirozzi, Prog. Polym. Sci., 1991, 16, 361.

24 Y. Zhou, J. Hu, B. Dang and J. He, RSC Adv., 2016, 6, 48720.

25 M. Pyda and B. Wunderlich, Macromolecules, 2005, 38, 10472.

26 S. Gogolewski and A. Pennings, J. Appl. Polym. Sci., 1983, 28, 1045.

27 H. Murase, Y. Ohta and T. Hashimoto, Macromolecules, 2011, 44, 7335.

28 P. Opaprakasit, M. Opaprakasit and P. Tangboriboonrat, Appl. Spectrosc., 2007, 61, 1352.

29 B. Na, N. Tian, R. Lv, Z. Li, W. Xu and Q. Fu, Polymer, 2010, 51, 563.

30 G. Kister, G. Cassanas and M. Vert, Polymer, 1998, 39, 267.

31 J. Zhang, Y. Duan, H. Sato, H. Tsuji, I. Noda, S. Yan and Y. Ozaki, Macromolecules, 2005, 38, 8012.

32 G. Stoclet, R. Seguela, J. M. Lefebvre, S. Elkoun and C. Vanmansart, Macromolecules, 2010, 43, 1488. 
33 J. Zhang, K. Tashiro, H. Tsuji and A. Domb, Macromolecules, 2007, 40, 1049.

34 K. Takahashi, D. Sawai, T. Yokoyama, T. Kanamoto and S. Hyon, Polymer, 2004, 45, 4969.

35 J. Zhang, K. Tashiro, H. Tsuji and A. Domb, Macromolecules, 2008, 41, 1352.

36 T. Cho and G. Strobl, Polymer, 2006, 47, 1036.

37 S. Lee, J. Han and J. Heo, Polymer, 2013, 54, 3624.
38 S. Lee, J. Han, Y. Jeong and M. Kwon, Macromolecules, 2009, 43, 25.

39 B. Wunderlich, Prog. Polym. Sci., 2003, 28, 383.

40 Y. Wang, M. Li and C. Shen, Mater. Lett., 2011, 65, 3525.

41 B. Na, N. Tian, R. Lv, Z. Li, W. Xu and Q. Fu, Polymer, 2010, 51, 563.

42 H. Xu, L. Xie, X. Jiang, M. Hakkarainen, J. B. Chen, G. J. Zhong and Z. M. Li, Biomacromolecules, 2014, 15, 1676. 